\title{
Cotinine-assessed second-hand smoke exposure and risk of cardiovascular disease in older adults
}

\author{
B J Jefferis, ${ }^{1}$ D A Lawlor, ${ }^{2}$ S Ebrahim, ${ }^{3}$ S G Wannamethee, ${ }^{1}$ C Feyerabend, ${ }^{4}$ \\ M Doig, ${ }^{4}$ L McMeekin, ${ }^{4}$ D G Cook, ${ }^{5}$ P H Whincup ${ }^{5}$
}

\begin{abstract}
- Additional data are published online only. To view these files please visit the journal online (http://heart.bmj.com).

${ }^{1} \mathrm{UCL}$ Department of Primary Care and Population Health, UCL Medical School, London, UK ${ }^{2}$ MRC CAiTE Centre, University of Bristol, Bristol, UK ${ }^{3}$ Non-Communicable Diseases Epidemiology Unit, Department of Epidemiology and Population Health, London School of Hygiene and Tropical Medicine, London, UK

${ }^{4}$ ABS Laboratories, BioPark, Welwyn Garden City, UK

${ }^{5}$ Division of Community Health Sciences, St George's, University of London, London, UK
\end{abstract}

\section{Correspondence to}

Dr B J Jefferis, UCL Department of Primary Care and Population Health, UCL Medical School, Rowland Hill Street, London NW3 2PF, UK:

b.jefferis@ucl.ac.uk

Accepted 25 March 2010

This paper is freely available online under the BMJ Journals unlocked scheme, see http:// heart.bmi.com/site/about/ unlocked.xhtml

\section{ABSTRACT}

Objectives To examine whether second-hand smoke (SHS) exposure measured by serum cotinine is associated with increased coronary heart disease (CHD) and stroke risk among contemporary older British adults. Design Prospective population-based study with selfreported medical history and health behaviours. Fasting blood samples were analysed for serum cotinine and cardiovascular disease (CVD) risk markers.

Setting Primary care centres in 25 British towns in 1998-2001.

Patients 8512 60-79-year-old men and women selected from primary care registers.

Main outcome measures Fatal and non-fatal myocardial infarction (Ml; $n=445)$ and stroke $(n=386)$ during median 7.8-year follow-up.

Main exposure Observational study of serum cotinine assayed from fasting blood sample using liquid chromatography tandem mass spectrometry method, and self-reported smoking history.

Results Among 5374 non-smokers without pre-existing CVD, geometric mean cotinine was $0.15 \mathrm{ng} / \mathrm{ml}$ (IOR $0.05-0.30)$. Compared with non-smokers with cotinine $\leq 0.05 \mathrm{ng} / \mathrm{ml}$, higher cotinine levels $(0.06-0.19$,

$0.2-0.7$ and $0.71-15.0 \mathrm{ng} / \mathrm{ml}$ ) showed little association with Ml; adjusted HRs were 0.92 (95\% $\mathrm{Cl} 0.63$ to 1.35), 1.07 (0.73 to 1.55$)$ and 1.09 (0.69 to 1.72$), p$ (trend) $=$ 0.69. Equivalent HRs for stroke were 0.82 (0.55 to 1.23), $0.74(0.48$ to 1.13$)$ and 0.69 (0.41 to 1.17$)$, p(trend) $=$ 0.065 . The adjustment for sociodemographic, behavioural and CVD risk factors had little effect on the results. The HR of MI for smokers (1-9 cigarettes/day) compared with non-smokers with cotinine $\leq 0.05 \mathrm{ng} / \mathrm{ml}$ was 2.14 (1.39 to 3.52) and 1.03 (0.52 to 2.04) for stroke.

Conclusions In contemporary older men and women, SHS exposure (predominantly at low levels) was not related to $\mathrm{CHD}$ or stroke risks, but we cannot rule out the possibility of modest effects at higher exposure levels.

\section{INTRODUCTION}

Second-hand tobacco smoke (SHS) exposure appears to increase the risks of coronary heart disease (CHD). Previous prospective studies of selfreported SHS exposure estimated the risk of $\mathrm{CHD}$ to be increased by 1.2- to 1.3-fold, independently of established CHD risk factors. ${ }^{1}{ }^{2}$ These studies were subsequently supported by the results of a large international case-control study of $\mathrm{CHD}$ risk factors $^{3}$ and by ecological studies of CHD mortality and morbidity rates in communities enacting bans on smoking in public places, which tended to find lower $\mathrm{CHD}$ rates after implementation of the ban. $^{4-7}$ Several cohort $^{8-10}$ or case-control ${ }^{11-13}$ studies report increased risks of ischaemic stroke associated with self-reported SHS exposure, though others did not identify significantly increased risks for new-onset stroke ${ }^{14}$ in non-smokers, or cerebrovascular mortality ${ }^{15}$ in never-smokers.

Self-reported SHS exposure is an imprecise exposure measure. A recent prospective study used serum cotinine, a stable metabolite of nicotine, as a marker of SHS exposure and found that high levels of SHS exposure (serum cotinine levels $>0.7 \mathrm{ng} / \mathrm{ml}$ ) may increase CHD risk by $1.4-$ to 1.5 fold. ${ }^{16}$ Moreover, a recent cross-sectional study based on data from the Third National Health and Nutrition Examination Survey (NHANES III) showed that low-level SHS exposure (cotinine levels as low as $0.2 \mathrm{ng} / \mathrm{ml}$ ) may influence inflammatory markers, though the association with CHD events was not studied. ${ }^{17}$ These studies suggest that the impact of SHS may have been underestimated in earlier studies and that large prospective studies using cotinine to examine the impact of SHS exposure on CHD and stroke events, particularly at lower cotinine levels and among populations with high CHD and stroke risk, are needed. We have therefore examined the associations between cotinine level, CHD and stroke in parallel studies of older British men and women who were initially studied in 1998-2001, when SHS exposures were markedly lower than those 20 years earlier. $^{18}$

\section{METHODS}

\section{Study design}

In 1998-2000, a total of 4252 men from a single general practice (primary care centre) in each of 24 British towns who were already participating in a prospective study of cardiovascular disease (CVD) attended for follow-up measurements at age $60-79$ years (77\% response rate). ${ }^{19}$ In $1999-2001$, a parallel study of 4286 women of the same age and in the same practices was established, with the addition of one study town (Bristol) and the omission of two others (Dewsbury and Maidstone) $\left(60 \%\right.$ response rate) ${ }^{20}$ Almost identical protocols for data collection were used. Ethical approval was provided by all relevant local research ethics committees. All participants provided written informed consent to the investigations, which were carried out in accordance with the Declaration of Helsinki. Participants completed questionnaires including detailed questions about current and previous smoking history (cigarettes, pipes and cigars), other health behaviours, occupation and medication use. Participants reported whether their spouse 
or partner smoked and if they currently lived alone or with their partner. Those cohabiting and reporting that the partner smoked were coded as exposed to SHS at home, while those cohabiting and reporting that the partner did not currently smoke, and those living alone were coded as not exposed to SHS at home. Nurses made physical measurements and collected fasting venous blood samples (see online supplementary data).

\section{Laboratory assays}

Serum samples were assayed for cotinine in 2007-8 at ABS Laboratories Ltd. For non-smokers a liquid chromatography tandem mass spectrometry assay with a lower limit of detection of $0.02 \mathrm{ng} / \mathrm{ml}$ and with a limit of quantification of $0.1 \mathrm{ng} / \mathrm{ml}$ was used. ${ }^{21}$ Cotinine values at the limit of quantification $(0.1 \mathrm{ng} / \mathrm{ml})$ were assigned a value of $0.05 \mathrm{ng} / \mathrm{ml}$, as in other studies, ${ }^{22}$ and statistical sensitivity analyses (using values of 0.025 and $0.075 \mathrm{ng} / \mathrm{ml}$ instead) assessed the validity of this assumption. Further details of the assay can be obtained from abslabs@biopark.org.uk. Assays for blood lipids and inflammatory markers are described in the online supplementary data.

\section{Classification of smokers and non-smokers}

Non-smokers reported no current cigarette, cigar or pipe smoking or any smoking in the past 5 years and had serum cotinine levels $\leq 15 \mathrm{ng} / \mathrm{ml}$, consistent with other literature. ${ }^{22}$ Among 7161 self-reported non-smokers, 7085 (99\%) had cotinine levels $\leq 15 \mathrm{ng} / \mathrm{ml}$, the remaining 76 (1\%) participants with cotinine $>15 \mathrm{ng} / \mathrm{ml}$ were recoded as smokers of $1-9$ cigarettes/ day, as their cotinine levels were similar to those of the other $(n=230)$ light smokers. No participants reported taking nicotine replacement therapy (British National Formulary code 4.10). ${ }^{23}$

\section{CHD and stroke outcome}

The outcome measures used were first fatal or non-fatal event of (a) myocardial infarction (MI) and (b) stroke occurring after the 1998-2000 survey (men) and 1999-2001 survey (women) and up to June 2008 (men) and September 2007 (women). Fatal cases were ascertained through the National Health Services Central Registers (death certificates with ICD-9 (International Classification of Diseases) codes 410-414 for MI and 430-438 for stroke and ICD 10 codes I21-I23, I252 for MI and I60, I61, I62, I629, I63-I66, I67, I672, I678, I679, I69, G450-453, G46 for stroke). Non-fatal events were recorded from 2-yearly reviews of patient primary care notes (including details of patient encounters with primary care and all correspondence and diagnoses from secondary care). MI was reported as heart attack or coronary thrombosis, diagnosed in accordance with WHO criteria; stroke was reported as cerebrovascular accident, cerebral thrombosis, haemorrhage or embolism.

\section{Statistical methods}

Serum cotinine was highly positively skewed and therefore analysed as a categorical variable. The threshold for the highest exposure group $(>0.7 \mathrm{ng} / \mathrm{ml})$ was chosen because cotinine $>0.7 \mathrm{ng} / \mathrm{ml}$ has been reported to be associated with increased $\mathrm{CHD}$ risks ${ }^{16}$ and because of the generally low cotinine levels in non-smokers in our study, there were too few participants to further subdivide those above this level. The lowest group had undetectable exposure $(\leq 0.05 \mathrm{ng} / \mathrm{ml})$ and participants with intermediate exposure were split into two equal-sized groups. Analyses were also completed with cotinine as a continuous variable that was log transformed (to base 2 ) to approximate normality.
Means, medians or proportions of behavioural and demographic factors selected a priori were calculated for groups of passive smokers (defined by cotinine level) and active smokers (defined by cigarettes/day). Linear trends across cotinine categories were tested using linear regression analyses with $\log _{2}$ cotinine as a predictor, to represent the effect of a doubling of cotinine concentration, adjusted for age, gender and region of residence. Skewed variables were natural log-transformed and adjusted for time of measurement if they showed significant diurnal variation. Blood pressure (BP), body mass index (BMI) and forced expiratory volume in $1 \mathrm{~s}\left(\mathrm{FEV}_{1}\right)$ were also adjusted for intraobserver variation. No diurnal or seasonal (October-March vs April-September) variation in cotinine levels was seen.

Cox proportional hazards regression models were used to estimate associations between serum cotinine and risk of $\mathrm{CHD}$ or stroke in non-smokers with complete data on covariates. Survival times were censored at date of MI or stroke, death from any cause, or end of follow-up period, whichever occurred first. Date of entry into the study was used as the time origin. The proportional hazards assumption was examined using timevarying covariates, calculating interactions of predictor variables and a function of survival time and including them in the models. Examination of time-varying covariates did not indicate violation of the proportionality assumption in the non-smoker sample. The HRs for categories of cotinine exposure compared with cotinine $\leq 0.05$ were estimated and the overall association in non-smokers was tested with the continuous association between $\log _{2}$ cotinine and CHD or stroke risk, adjusted for gender, age (continuous variable) and region of residence. Models were adjusted for covariates associated with both CHD risk and SHS, first established biological risk factors and inflammatory markers as continuous variables: systolic and diastolic BP, total and high-density lipoprotein cholesterol, BMI, FEV 1 , natural log triglycerides and white cell count, C-reactive protein (CRP) and interleukin 6 (IL-6). Models were then further adjusted for preexisting diabetes (yes/no) and behavioural risk factors as categorical variables: physical activity (inactive $(<3 \mathrm{~h}$ moderate or vigorous activity/week) or active), alcohol intake (none/occasional, light, heavy), social class (I and II, III non-manual, III manual, IV and V manual and armed forces). Interactions with gender and age were tested using likelihood ratio (LR) tests. In a sensitivity analysis, regression models excluded past smokers from the non-smoker group. All hypothesis tests were two sided and significance levels are reported.

\section{RESULTS}

Among 8512 participants (4267 women, 4245 men), 7375 had questionnaire data on cigarette smoking and cotinine data Participants with a history (self-report or medical record) of $\mathrm{MI}$ or stroke $(n=1168)$, current smokers of $\geq 10$ cigarettes/day $(n=276)$ and recent ex-smokers (within 5 years) $(n=251)$ were excluded from analyses. The analysis sample included 5374 nonsmokers (of whom 2783, 52\% were never-smokers) and, as a comparison group, 306 smokers of 1-9 cigarettes/day. In the analysis sample of 5680 participants, there were 217 new MI cases (192 in non-smokers) and 176 new stroke cases (165 in non-smokers) in mean follow-up times of 8.3 years (men) and 7.0 years (women). Geometric mean cotinine level was $104.57 \mathrm{ng} / \mathrm{ml}$ (IOR 66-227) in active smokers (1-9 cigarettes/ day) and $0.15 \mathrm{ng} / \mathrm{ml}$ (IOR 0.05-0.30) in non-smokers ( $\mathrm{p}$ no difference $<0.001)$. Of the 5374 non-smokers, $2256(42 \%)$ had undetectable cotinine levels $(\leq 0.05 \mathrm{ng} / \mathrm{ml}$ ). Table 1 shows the characteristics of SHS-exposed non-smokers and of active 
Table 1 Association between serum cotinine in non-smokers ( $\geq 5$ years since quitting) or active smokers and cardiovascular disease risk factors in men and women (mean (SD) or \%(n))*

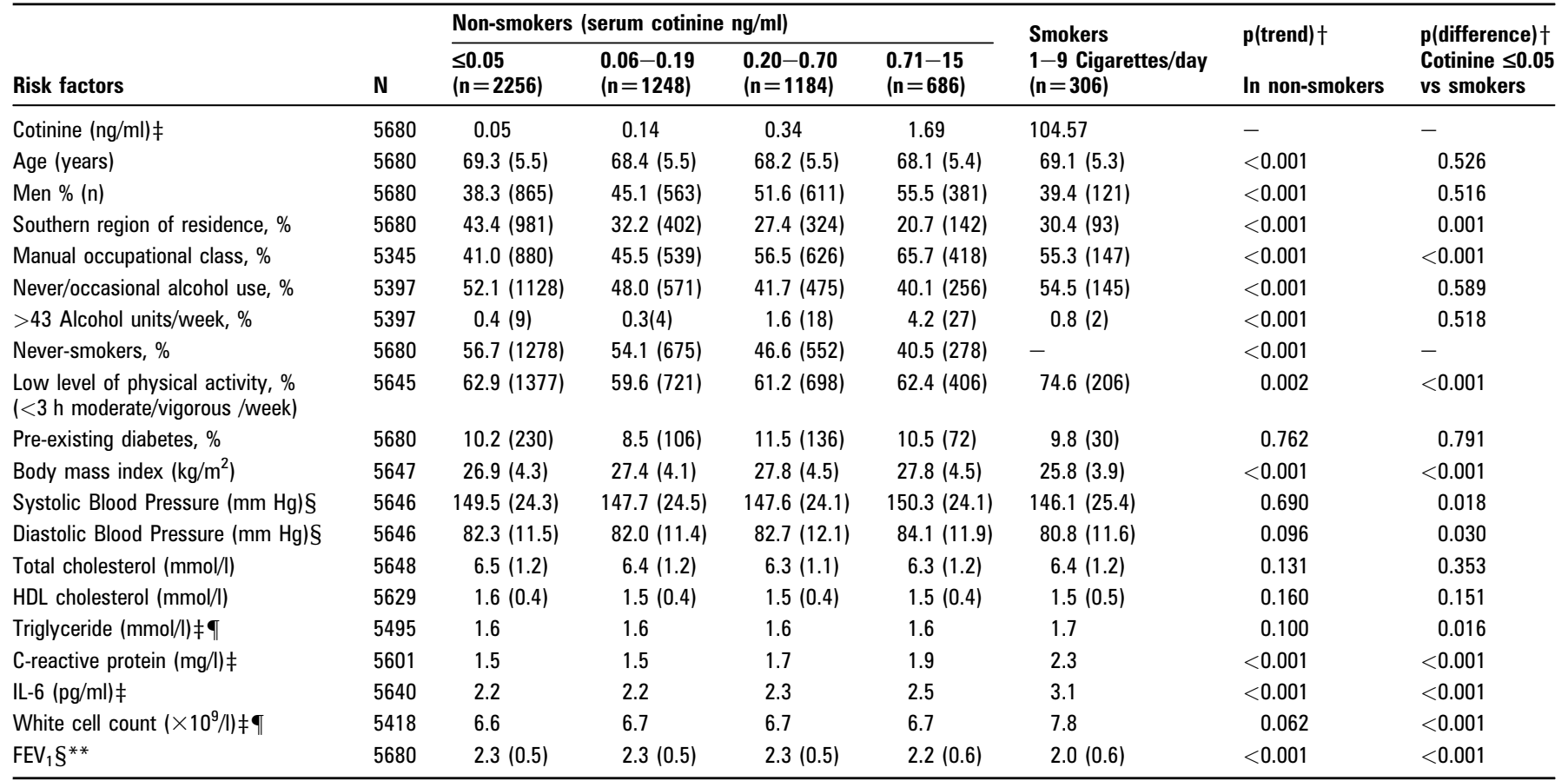

${ }^{*}$ Non-smoker sample excludes (i) current cigarette, pipe or cigar smokers, (ii) ex-smokers who quit $<5$ years ago, (iii) pre-existing myocardial infarction or stroke. Means are adjusted for age, gender and region;

$t p$ (trend) from linear regression models of In(cotinine) in non-smokers, $p$ (difference) from linear regression models contrasting cotinine $<0.05$ with $1-9 /$ day smokers. Models adjusted for age gender and region;

łgeometric mean;

§adjusted for interobserver variation;

TAdjusted for time of day;

**adjusted for height squared and age.

$\mathrm{FEV}_{1}$, forced expiratory volume in $1 \mathrm{~s}$; HDL, high-density lipoprotein; IL, interleukin.

smokers, both compared with the baseline group of non-smokers with undetectable SHS exposure (cotinine $\leq 0.05 \mathrm{ng} / \mathrm{ml}$ ). Active smokers tended to be resident in the north of the UK, from manual social class and physically inactive compared with nonsmokers with undetectable SHS exposure. Non-smokers with higher serum cotinine levels, indicating greater SHS exposure, were also resident in the north of the UK, from manual social class, physically inactive and younger, male, ex-smokers rather than never-smokers, and less likely to be non-drinkers or occasional alcohol drinkers compared with non-smokers with undetectable SHS exposure. Among non-smokers, cotinine level was positively and significantly associated with higher CRP, IL-6 and BMI and with lower $\mathrm{FEV}_{1}$. Blood pressure, lipids and preexisting diabetes showed no association with cotinine. Active smokers, had lower BMI, systolic BP, diastolic BP and $\mathrm{FEV}_{1}$ but higher triglycerides, CRP, IL-6 and white cell count than nonsmokers with undetectable SHS exposure.

In Cox proportional hazard models examining the association between cotinine level and CHD risk among non-smokers and adjusted for age, gender and region of residence (table 2, model 1), a doubling of cotinine level was not strongly associated with CHD risk: HR 1.02 (95\% CI 0.95 to 1.11), $\mathrm{p}=0.56$. Increasing cotinine categories showed no appreciable association with CHD risk when compared with participants with cotinine $\leq 0.05 \mathrm{ng} / \mathrm{ml}$. Further adjustments for established cardiovascular risk factors and inflammatory markers (model 2) and preexisting diabetes, social class and behavioural risk factors (model 3) did not materially alter the results. LR tests showed no evidence for interactions of cotinine on risk of MI by gender $(p=0.94)$ or age $(p=0.65)$. Exclusion of ex-smokers did not materially affect the results. In contrast, active smokers, when compared with the same reference group (non-smokers with cotinine $\leq 0.05 \mathrm{ng} / \mathrm{ml}$ ) had a HR for MI of 2.53 (95\% CI 1.61 to 3.99 ), reducing slightly to 2.14 (95\% CI 1.39 to 3.52$)$ after adjustment for established risk factors (equivalent to model 3 in table 2).

In Cox proportional hazard models examining the association between cotinine level and stroke risk among non-smokers and adjusted for age, gender and region of residence (table 3 , model 1), increasing cotinine levels showed a weak inverse association with stroke risk compared with participants with cotinine $\leq 0.05 \mathrm{ng} / \mathrm{ml}$. A doubling of cotinine level was associated with a decrease in stroke risk of 0.91 (95\% CI 0.83 to 1.01), $\mathrm{p}=0.07$. Further adjustments for established cardiovascular risk factors, social class and inflammatory markers (models 2 and 3) did not materially alter the results. LR tests showed no evidence for interactions of cotinine on risk of stroke by gender $(p=0.92)$ or age $(p=0.83)$. Exclusion of ex-smokers did not materially affect the results. Active smokers, when compared with the same reference group (non-smokers with cotinine $\leq 0.05 \mathrm{ng} / \mathrm{ml}$ ) had a HR for stroke of 1.13 (95\% CI 0.60 to 2.13), reducing to 1.03 (95\% CI 0.52 to 2.04 ) after adjustment (equivalent to model 3 in table 3).

Analyses presented were not influenced either by the cotinine threshold used to define active smoking (use of a more conservative cotinine threshold $(9.5 \mathrm{ng} / \mathrm{ml})^{24}$ did not materially affect results), or by the duration of follow-up period (HRs did not differ between early and late follow-up periods).

Among participants with data on self-reported exposure to SHS in the home $(n=4818), 485(10 \%)$ lived with a spouse/ 
Table 2 Hazard ratios (HRs) for risk of myocardial infarction (MI) in men and women by serum cotinine or active smoking level

\begin{tabular}{|c|c|c|c|c|c|c|c|}
\hline & \multicolumn{4}{|c|}{ Non-smokers (serum cotinine $\mathrm{ng} / \mathrm{ml})(\mathrm{n}=4608)^{*}$} & \multirow{2}{*}{$\begin{array}{l}\text { Smokers }\left(n=242^{*}\right) \\
1-9 \text { Cigarettes/day }\end{array}$} & \multicolumn{2}{|l|}{ All non-smokers } \\
\hline & $\leq 0.05$ & $0.06-0.19$ & $0.20-0.70$ & $0.71-15$ & & Cotinine $\leq 15 \mathrm{ng} / \mathrm{ml} \dagger$ & $\mathbf{p}$ (trend) $\dagger$ \\
\hline Mean cotinine (ng/ml) & 0.05 & 0.14 & 0.34 & 1.71 & 100.02 & 0.15 & \\
\hline Number of participants & 1943 & 1087 & 1018 & 560 & 242 & 4608 & \\
\hline Person-years & 14641 & 8323 & 7902 & 4333 & 1701 & 35199 & \\
\hline CHD rates/1000 & 5.26 & 4.81 & 5.95 & 6.46 & 14.67 & 5.45 & \\
\hline \multicolumn{8}{|l|}{$\mathrm{HR}(95 \% \mathrm{Cl})$} \\
\hline Model 3 & 1.00 & 0.92 (0.63 to 1.35$)$ & 1.07 (0.73 to 1.55$)$ & 1.09 (0.69 to 1.72$)$ & 2.14 (1.39 to 3.52$)$ & $1.02(0.94$ to 1.11$)$ & 0.690 \\
\hline Excluding former smokers & & & $(n=2321)$ & & $(n=239)$ & & \\
\hline Mean cotinine (ng/ml) & 0.05 & 0.14 & 0.33 & 1.76 & 100.02 & 0.13 & \\
\hline Number of participants & 1081 & 572 & 458 & 210 & 239 & 2321 & \\
\hline Number of events & 34 & 17 & 16 & 7 & 25 & 74 & \\
\hline Model 2 & 1.00 & $0.90(0.49$ to 1.60$)$ & $0.98(0.53$ to 1.81$)$ & $0.95(0.412 .20)$ & 2.10 (1.17 to 3.76$)$ & $1.00(0.87$ to 1.16$)$ & 0.985 \\
\hline Model 3 & 1.00 & 0.91 (0.50 to 1.63 ) & 0.99 (0.53 to 1.84$)$ & 0.94 (0.40 to 2.24$)$ & $2.17(1.20$ to 3.90$)$ & 1.00 (0.86 to 1.16 ) & 0.975 \\
\hline
\end{tabular}

*HRs for non-smokers from models with cotinine $\leq 0.05$ as baseline. HRs for smokers from models comparing cotinine $\leq 0.05$ with smokers of $1-9$ cigarettes/day. Participants with preexisting myocardial infarction are excluded.

†From Cox regression models of $\log _{2}$ (cotinine) level (representing a doubling in cotinine) and CHD in non-smokers, cotinine $\leq 15 \mathrm{ng} / \mathrm{ml}$.

Model 1 = age + gender + region; Model $2=$ model $1+\mathrm{BMI}+\mathrm{SBP}+\mathrm{DBP}+$ total cholesterol $+\mathrm{HDL}+$ triglycerides $+\mathrm{FEV}+\mathrm{CRP}+\mathrm{IL}-6+$ white cell count; $\mathrm{Model} 3=$ model $2+$ pre-existing

diabetes + physical activity (inactive or active) + alcohol intake (none/occasional, light, heavy) + smoking history (never/ time since quitting) + social class (non-manual, manual and armed forces)

$\mathrm{CHD}$, coronary heart disease; CRP, C-reactive protein; FEV 1 , forced expiratory volume in $1 \mathrm{~s}$; HDLc, high-density lipoprotein cholesterol; IL, interleukin.

partner who smoked and $4333(90 \%)$ did not live with a partner who smoked; geometric mean cotinine in these groups were respectively $0.13 \mathrm{ng} / \mathrm{ml}$ (IOR $0.05-0.25$ ) and $0.70 \mathrm{ng} / \mathrm{ml}$ (IOR $0.22-2.4)$. Non-smoking participants who lived with a smoker had a HR for CHD of 1.33 (95\% CI 0.82 to 2.15) and a HR for stroke of 0.53 (95\% CI 0.25 to 1.16 ) compared with those not living with a smoker, adjusted for age, gender and region. Further adjustments for established cardiovascular risk factors, social class and inflammatory markers did not materially alter the results and there was no evidence of effect modification by gender or age. Restricting analyses to never-smokers, in the sample with full covariate data: 110 (7\%) lived with a smoker and 1457 (93\%) did not, geometric mean cotinine levels were respectively $0.12 \mathrm{ng} / \mathrm{ml}$ (IOR $0.05-0.21$ ) and $0.74 \mathrm{ng} / \mathrm{ml}$ (IOR 0.25-2.46). Never-smoking participants who lived with a smoker compared with those who did not live with a smoker

Table 3 Hazard ratios (HRs) for risk of stroke in men and women by serum cotinine or active smoking level

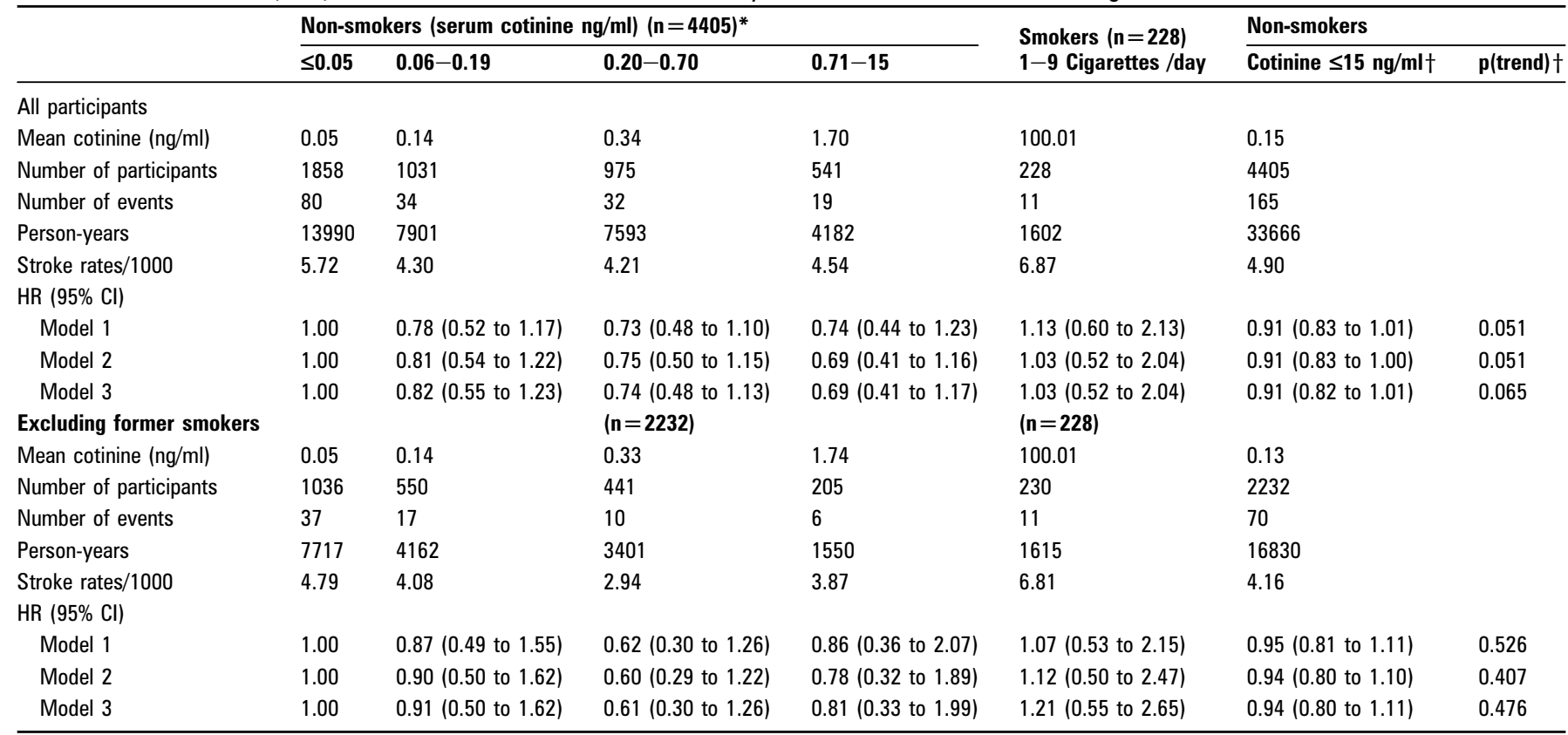

*HRs for non-smokers from models with cotinine $\leq 0.05$ as baseline. HRs for smokers from models comparing cotinine $\leq 0.05$ with smokers of $1-9$ cigarettes/day. Participants with preexisting myocardial infarction or stroke are excluded.

†From Cox regression models of $\log _{2}$ (cotinine) level (representing a doubling in cotinine) and stroke in non-smokers, cotinine $\leq 15 \mathrm{ng} / \mathrm{ml}$.

Model 1 = age + gender + region; Model $2=$ model $1+\mathrm{BMI}+\mathrm{SBP}+\mathrm{DBP}+$ total cholesterol $+\mathrm{HDL}+$ triglycerides $+\mathrm{FEV}+\mathrm{CRP}+\mathrm{IL}-6+$ white cell count; Model $3=$ model $2+$ pre-existing

diabetes + physical activity (inactive or active) + alcohol intake (none/occasional, light, heavy) + smoking history (never/ time since quitting) + social class (non-manual, manual and armed forces) CHD, coronary heart disease; CRP, C-reactive protein; FEV 1 , forced expiratory volume in $1 \mathrm{~s}$; HDLc, high-density lipoprotein cholesterol; IL, interleukin. 
had an age, gender and region adjusted HR for CHD of 2.17 (95\% CI 0.97 to 4.84 ) strengthening to 2.41 (95\% CI 1.04 to 5.59 ) on full adjustment, and a fully adjusted HR for stroke of 0.69 (95\% CI 0.16 to 2.94). There was no evidence for effect modification by age, although data were too sparse to estimate interactions with gender.

\section{DISCUSSION \\ Main findings}

In this population of older men and women with low levels of SHS exposure (indicated by low mean levels of serum cotinine among non-smokers and also the small prevalence (just 10\%) of self-report of living with a partner who smoked) there was little evidence for an association between SHS exposure and either MI or stroke, irrespective of whether these were never-smokers or ex-smokers. These findings did not vary by age or by gender. However, there was evidence of a raised risk of MI in neversmokers who lived with a smoker, though the confidence limits are wide and this was a post hoc analysis.

\section{Relation to earlier studies}

Previous studies and meta-analyses have shown that SHS exposure, particularly defined by partner smoking, is associated with a $25-30 \%$ increase in risk of $\mathrm{CHD}^{1}{ }^{2}{ }^{25}$ an estimate recently supported by the INTERHEART Study. ${ }^{3}$ Evidence from cotinine-based studies suggests that the increase in CHD risk at high overall SHS exposure (particularly at exposure levels associated with cotinine concentrations $>0.7 \mathrm{ng} / \mathrm{ml}$ ) may exceed these earlier estimates, and be as high as $45-50 \%{ }^{16}$ Subsequently, cross-sectional studies relating cotinine levels to intermediate markers such as fibrinogen and white cell count have suggested that the effects of cotinine on cardiovascular risk might begin at SHS exposure levels associated with lower cotinine concentrations $(<0.7 \mathrm{ng} / \mathrm{ml}) .{ }^{17} 26$

Our investigation is the first to provide data on the association of both CHD and stroke risk with lower cotinine levels $(<0.7 \mathrm{ng} / \mathrm{ml})$ resulting from lower nicotine exposure due to public health measures. The low overall SHS exposure levels (24\% of non-smokers have cotinine levels between 0.06 and $0.70 \mathrm{ng} / \mathrm{ml}$ ) mean our cohorts are well placed to provide information about any continued cardiovascular risk in contemporary groups with low SHS exposure. In line with a case-control study based on self-reported lifetime SHS exposure in the context of falling SHS exposure levels, ${ }^{27}$ our results provide little support for an increased risk of CHD or stroke associated with low levels of cotinine exposure. However, we are unable to exclude adjusted HRs for CHD as high as 1.53 , when comparing cotinine levels between 0.06 and $0.70 \mathrm{ng} / \mathrm{ml}$ with cotinine $\leq 0.05 \mathrm{ng} / \mathrm{ml}$. We had limited information on the effects of higher SHS exposure, associated with cotinine levels $0.71-15 \mathrm{ng} / \mathrm{ml}$; only $13 \%$ of non-smokers had a cotinine level in this range. Higher SHS exposure appeared to be associated with a very modest increase in CHD risk and could only be imprecisely estimated. The results are, however, consistent with the increase in CHD risk of $45 \%$ associated with such cotinine levels in our earlier study, although we reported even greater elevations in risk over shorter follow-up periods. ${ }^{16}$

The pattern of overall association between cotinine level and $\mathrm{CHD}$ risk among non-smokers in this study (expressed as a $2 \%$ $(-5 \%$ to $11 \%)$ increase in CHD risk for each doubling of cotinine concentration) is weaker than in the previous study, where the equivalent estimate was $16 \%$ ( $6 \%$ to $27 \%) .{ }^{16}$ This study population is appreciably older than in the previous study and includes both genders. Although within this study neither gender nor age appreciably influenced cotinine-CHD associations, the relative strengths of associations between many major risk markers (especially active smoking) and CHD are recognised to attenuate with increasing age, ${ }^{28} 29$ so our estimates may be lower than estimates which would be observed in a middle-aged population. The point estimate of the effect of partner smoking on CHD risk in never-smokers is considerably stronger than in the published literature, while the association with stroke is weaker. ${ }^{1}$ Both estimates were post hoc and based on small numbers and had wide confidence limits.

The literature for stroke is less conclusive than for CHD and fewer previous reports exist. Two case-control studies ${ }^{11} 12$ and three prospective cohort studies ${ }^{8-10}$ reported positive associations between self-reported SHS exposure and stroke risk, although not all studies do. One prospective cohort had limited power to detect an effect on cerebrovascular mortality as distinct from cardiovascular mortality ${ }^{15}$ and another had few cases of stroke among non-smoking women. ${ }^{14}$ Other studies did not find significantly elevated risks of subarachnoid haemorrhage $^{30}$ or silent cerebral infarction ${ }^{31}$ associated with SHS exposure from living with a spouse who smokes. Our previous prospective study showed no evidence of an association between cotinine level in non-smokers and stroke risk. In this study, no association was found between stroke and SHS exposure measured by cotinine, over a very wide range of exposure (cotinine from 0.05 to $15 \mathrm{ng} / \mathrm{ml}$ ), or measured by living with a spouse who smoked. However, our analyses have limited precision and cannot exclude the possibility of a moderately strong association. The lack of association with stroke is consistent with our finding in this population of no association of cotinine with variation in BP, an association that was precisely estimated (table 1). By contrast, cotinine was associated with several CHD risk factors, including inflammatory markers, despite not showing a strong association with CHD events. We did not observe the expected associations between active smoking and stroke, perhaps because we only studied smokers of 1-9 cigarettes/day and not heavier smokers.

\section{Strengths and weaknesses}

A key strength of this study is the use of serum cotinine as an objective measure of SHS exposure. Cotinine is the major metabolite $(70-80 \%)$ of nicotine, reflecting recent overall SHS exposure, which enables more accurate estimates of the associations between SHS exposure and CVD than the use of selfreported exposures, which may only partially capture exposures from different sources. ${ }^{32} 33$ Nicotine is metabolised into cotinine by cytochrome P450. Although genetic factors controlling nicotine metabolism may influence cotinine levels, ${ }^{34}$ unless these are systematically associated with CHD or stroke risk (for which there is little current evidence), they should not bias the estimation of cotinine-CHD associations.

While it is possible that other environmental influences on cotinine levels exist, the evidence for them is weak at the present time and we have included adjustment for BMI and alcohol intake in our models, which did not have any important effect. Cotinine is well validated as a biomarker of nicotine exposure ${ }^{33}$ and also prevents appreciable misclassification of active smokers who fail to self-report smoking. We reclassified 76 participants who reported being non-smokers but had cotinine levels $>15 \mathrm{ng} / \mathrm{ml}$ (a standard cut-off point) as active smokers. Including the 76 participants in the analysis in the highest exposure group did not materially alter any of our results. This study was large, combining cohorts of older men and women from socially and geographically representative population studies. However, the 
removal of a substantial number of subjects with pre-existing disease and low event rates limited the statistical power of the study to detect small but important elevations in CVD risk. The results are based entirely on older populations of retirement age, exposed to contemporary SHS levels and at relatively high CVD risk and may not be applicable to younger subjects or to populations with higher SHS exposure. In particular, this older population is less likely to be exposed to SHS in the workplace and may be less likely to be exposed in leisure public places than younger populations.

\section{CONCLUSIONS}

The results suggest that the lower levels of cotinine prevailing in the UK in 1998-2001, even before the introduction of legislation to reduce SHS exposure in public places, were not apparently associated with any marked increase in CHD or stroke risk in older people. Our findings relating to low levels of SHS exposure do not undermine previous epidemiological studies reporting positive associations between SHS exposure and CHD or stroke events, or the weight of laboratory studies suggesting causal associations between SHS and elevated CHD risk. However, because of the limited statistical power of our study and the older age range of the population, further evidence from pooled analyses of large population studies, including people of working age, are needed to establish the associations between low-level SHS exposure and CHD risk. In addition, studies specifically examining the continuing impact of domestic partner smoking, which is the main determinant of high SHS exposure in this population, ${ }^{18}$ would be valuable.

Acknowledgements We acknowledge Mary Thomas, Andrew Thomson, Lucy Lennon, Claire Carson and Antoinette Amuzu for data management.

Funding This work was supported by the British Heart Foundation: the British Regional Heart Study Research Group is supported by (programme grant RG/04/003). Cotinine analyses were funded by (project grant PG/06/154/22043). The British Women's Heart and Health Study is jointly funded by the UK Department of Health and the British Heart Foundation. The views expressed in this publication are those of the authors and not necessarily those of the funding bodies.

Competing interests None.

Ethics approval This study was conducted with the approval of the London Multicentre Research Ethics Committee.

Provenance and peer review Not commissioned; externally peer reviewed.

\section{REFERENCES}

1. He J, Vupputuri S, Allen $\mathrm{K}$, et al. Passive smoking and the risk of coronary heart disease-a meta-analysis of epidemiologic studies. N Engl J Med 1999;340:920-6.

2. Barnoya J, Glantz SA. Cardiovascular effects of secondhand smoke: nearly as large as smoking. Circulation 2005;111:2684-98

3. Teo KK, Ounpuu S, Hawken S, et al. Tobacco use and risk of myocardial infarction in 52 countries in the INTERHEART study: a case-control study. Lancet 2006;368:647-58.

4. Pell JP, Haw S, Cobbe S, et al. Smoke-free legislation and hospitalizations for acute coronary syndrome. N Engl J Med 2008;359:482-91.

5. Cesaroni G, Forastiere F, Agabiti N, et al. Effect of the italian smoking ban on population rates of acute coronary events. Circulation 2008;117:1183-8.

6. Juster HR, Loomis BR, Hinman TM, et al. Declines in hospital admissions for acute myocardial infarction in new york state after implementation of a comprehensive smoking ban. Am J Public Health 2007:97:2035-9.
7. Meyers DG, Neuberger JS, He J. Cardiovascular effect of bans on smoking in public places: a systematic review and meta-analysis. J Am Coll Cardiol 2009;54:1249-55.

8. Glymour MM, Defries TB, Kawachi I, et al. Spousal smoking and incidence of first stroke: the health and retirement study. Am J Prev Med 2008:35:245-8.

9. Zhang X, Shu XO, Yang G, et al. Association of passive smoking by husbands with prevalence of stroke among chinese women nonsmokers. Am J Epidemiol 2005;161:213-18.

10. Iribarren C, Darbinian J, Klatsky AL, et al. Cohort study of exposure to environmental tobacco smoke and risk of first ischemic stroke and transient ischemic attack. Neuroepidemiology 2004;23:38-44.

11. Bonita R, Duncan J, Truelsen T, et al. Passive smoking as well as active smoking increases the risk of acute stroke. Tob Control 1999:8:156-60.

12. You RX, Thrift AG, McNeil JJ, et al. Ischemic stroke risk and passive exposure to spouses' cigarette smoking. Melbourne stroke risk factor study (MERFS) group. Am J Public Health 1999:89:572-5.

13. He Y, Lam TH, Jiang $B$, et al. Passive smoking and risk of peripheral arterial disease and ischemic stroke in chinese women who never smoked. Circulation 2008;118:1535-40.

14. Qureshi Al, Suri MF, Kirmani JF, et al. Cigarette smoking among spouses: anothe risk factor for stroke in women. Stroke 2005;36:e74-76.

15. Hill SE, Blakely T, Kawachi I, et al. Mortality among lifelong nonsmokers exposed to secondhand smoke at home: cohort data and sensitivity analyses. Am J Epidemiol 2007:165:530-40.

16. Whincup PH, Gilg JA, Emberson JR, et al. Passive smoking and risk of coronary heart disease and stroke: prospective study with cotinine measurement. $B M J$ 2004:329:200-5

17. Venn A, Britton J. Exposure to secondhand smoke and biomarkers of cardiovascular disease risk in never-smoking adults. Circulation 2007:115:990-5.

18. Jefferis BJ, Thomson AG, Lennon LT, et al. Changes in environmental tobacco smoke (ETS) exposure over a 20-year period: cross-sectional and longitudinal analyses. Addiction 2009:104:496-503.

19. Walker M, Whincup PH, Shaper AG. The British Regional Heart Study 1975-2004 Int J Epidemiol 2004;33:1185-92.

20. Lawlor DA, Bedford C, Taylor M, et al. Geographical variation in cardiovascular disease, risk factors, and their control in older women: British Women's Heart and Health Study. J Epidemiol Community Health 2003;57:134-40.

21. Feyerabend C, Russell MA. A rapid gas-liquid chromatographic method for the determination of cotinine and nicotine in biological fluids. J Pharm Pharmacol 1990:42:450-2.

22. Jarvis MJ, Feyerabend C, Bryant $A$, et al. Passive smoking in the home: plasma cotinine concentrations in non-smokers with smoking partners. Tob Control 2001;10:368-74

23. Joint Formulary Committee. British national formulary. 38 edn. London: British Medical Association and Royal Pharmaceutical Society of Great Britain, 1999.

24. Jarvis MJ, Fidler J, Mindell J, et al. Assessing smoking status in children, adolescents and adults: cotinine cutpoints revisited. Addiction 2008;103:1553-61.

25. Law MR, Morris JK, Wald NJ. Environmental tobacco smoke exposure and ischaemic heart disease: an evaluation of the evidence. BMJ 1997;315:973-80.

26. Jefferis BJ, Lowe GD, Welsh P, et al. Secondhand smoke (SHS) exposure is associated with circulating markers of inflammation and endothelial function in adult men and women. Atherosclerosis 2010;208:550-6.

27. Stranges S, Bonner MR, Fucci F, et al. Lifetime cumulative exposure to secondhand smoke and risk of myocardial infarction in never smokers: results from the Western New York Health Study, 1995-2001. Arch Intern Med 2006;166:1961-7.

28. Lewington S, Whitlock G, Clarke R, et al. Blood cholesterol and vascular mortality by age, sex, and blood pressure: a meta-analysis of individual data from 61 prospective studies with 55000 vascular deaths. Lancet 2007;370:1829-39.

29. Lewington S, Clarke R, Qizilbash N, et al. Age-specific relevance of usual blood pressure to vascular mortality: a meta-analysis of individual data for one million adults in 61 prospective studies. Lancet 2002;360:1903-13.

30. Anderson CS, Feigin V Bennett D et al. Active and passive smoking and the risk of subarachnoid hemorrhage: an international population-based case-control study. Stroke 2004;35:633-7.

31. Howard G, Wagenknecht LE, Cai J, et al. Cigarette smoking and other risk factors for silent cerebral infarction in the general population. Stroke 1998;29:913-17.

32. Kemmeren JM, Vanpoppel G, Verhoef $P$, et al. Plasma cotinine: stability in smokers and validation of self-reported smoke exposure in nonsmokers. Environmental Research 1994;66:235-43.

33. Benowitz NL. Cotinine as a biomarker of environmental tobacco smoke exposure Epidemiol Rev 1996;18:188-204.

34. Benowitz NL. Clinical pharmacology of nicotine: implications for understanding, preventing, and treating tobacco addiction. Clin Pharmacol Ther 2008:83:531-41. 